\title{
Effect of specific cathode surface area on biofouling in an anaerobic electrochemical membrane bioreactor: novel insights using high- speed video camera
}

Veerraghavulu Sapireddy ${ }^{\mathrm{a}}$, Ala'a Ragab ${ }^{\mathrm{a}}$, Krishna P. Katuri ${ }^{\mathrm{a}}$, Yuanlie Yu ${ }^{\mathrm{b}}$, Zhiping Lai ${ }^{\mathrm{b}}$, Erqiang Li $^{\mathrm{c}}$, Sigurdur T. Thoroddsen ${ }^{\mathrm{c}}$, Pascal E. Saikaly ${ }^{\mathrm{a}, *}$

${ }^{a}$ Biological and Environmental Sciences and Engineering Division, Water Desalination and Reuse Research Center, King Abdullah University of Science and Technology, Thuwal 23955-6900, Saudi Arabia

${ }^{\mathrm{b}}$ Advanced Membranes and Porous Materials Research Center, King Abdullah University of Science and Technology, Thuwal 23955-6900, Saudi Arabia

'Division of Physical Sciences and Engineering, King Abdullah University of Science and Technology (KAUST), Thuwal 23955-6900, Saudi Arabia

*Corresponding author: Biological and Environmental Sciences and Engineering Division, Water Desalination and Reuse Research Center, King Abdullah University of Science and Technology, Thuwal 23955-6900, Saudi Arabia.

Email address: Pascal.saikaly@kaust.edu.sa (P.E. Saikaly)

\footnotetext{
ABSTRACT

The effect of the specific cathode surface area (SCSA; 2, 4 and $8 \mathrm{~m}^{2} / \mathrm{m}^{3}$ ) on biogas production and biofouling control in anaerobic electrochemical membrane bioreactors (AnEMBRs) was investigated. Nickel-based hollow fibers were used as both a cathode for hydrogen evolution and membrane for effluent filtration. Varying the SCSA was found to influence the current density, biogas quantity and composition, and trans-membrane pressure (TMP). In situ-gas generation was monitored using a high-speed camera; where the $8 \mathrm{~m}^{2} / \mathrm{m}^{3}$ AnEMBRs were found to mainly evolve smaller sized bubbles $(45-114 \mu \mathrm{m})$ compared to the 2 and $4 \mathrm{~m}^{2} / \mathrm{m}^{3}$ AnEMBRs. This correlated with the lowest TMP $(0.37$ bars $)$ compared to the other reactors $\left(4 \mathrm{~m}^{2} / \mathrm{m}^{3} ; 0.76\right.$ bars, $2 \mathrm{~m}^{2} / \mathrm{m}^{3} ; 1.18$ bars). The cathodic microbial community varied with SCSA, with Acetobacterium dominating at a relative abundance that reached $62 \%\left(4 \mathrm{~m}^{2} / \mathrm{m}^{3}\right)$, while hydrogenotrophic methanogens (represented by Methanocorpusculum) comprised up to $2.5 \%$ of the total
} 
community. This community variability apparently influenced both the hydrogen $\left(\mathrm{Q}_{\mathrm{H} 2}\right)$ and methane $\left(\mathrm{Q}_{\mathrm{CH} 4}\right)$ production rates, reaching a maximum $\mathrm{Q}_{\mathrm{H} 2}$ of $0.416 \mathrm{~m}^{3} / \mathrm{m}^{3} /$ day $\left(\mathrm{SCSA} 8 \mathrm{~m}^{2} / \mathrm{m}^{3}\right)$.

Overall, this study discloses the cumulative effect of $\mathrm{Q}_{\mathrm{H} 2}, \mathrm{Q}_{\mathrm{CH} 4}$, bubble distribution and frequency on biofouling with SCSA variation in AnEMBRs, demonstrating that with higher SCSA the in-situ gas scouring effect is more pronounced leading to a lower fouling propensity.

Keywords: electrochemical membrane bioreactor; biofouling; microbial electrolysis cell; specific cathode surface area; hydrogen gas scouring

\author{
Abbreviations \\ AnEMB: Anaerobic electrochemical membrane bioreactor \\ CE: Coulombic efficiency \\ $\mathrm{CH}_{4}$ : Methane \\ $\mathrm{H}_{2}$ : Hydrogen \\ HF: Hollow fibers \\ Hz: Frequency \\ MBR: Membrane bioreactor \\ MEC: Microbial electrolysis cell \\ Ni-HFM: Nickel-hollow fiber membrane \\ Pdf: Probability density distribution \\ $\mathrm{QH}_{2}$ : Hydrogen production rate \\ $\mathrm{Q}_{\mathrm{CH} 4}$ : Methane production rate \\ $\mathrm{r}_{\mathrm{Cat}} \mathrm{H}_{2}$ : Cathodic recovery of hydrogen \\ $\mathrm{r}_{\mathrm{Cat}} \mathrm{CH}_{4}$ : Cathodic recovery of methane \\ SCSA: Specific cathode surface area \\ SEM: Scanning electron microscopy \\ TMP: Trans-membrane pressure"
}

\title{
1. Introduction
}

Wastewater is currently viewed as a valuable resource of water, energy, nutrients (nitrogen and phosphorous) and materials [1]. In this regard, anaerobic biological processes such as microbial electrolysis cells (MECs) offer an opportunity to biologically treat wastewater with the concomitant recovery of energy [2]. In MEC, microorganisms with extracellular electron transfer capability transfer the electrons generated during the oxidation of organics in wastewater to the anode. The electrons and protons generated at the anode during oxidation are utilized at the 
cathode for the production of $\mathrm{H}_{2}$ [3]. Additional voltage (0.2-0.8 V) lower than the voltage required for traditional water electrolysis (1.8-3.5 V) must be applied between the electrodes [4]. However, MECs alone cannot produce high-quality water suitable for reuse, for that purpose they have to be integrated with membrane bioreactors (MBRs). A number of studies have reported the integration of MECs with MBRs [2, 5-7], in what is referred to as anaerobic electrochemical membrane bioreactor (AnEMBR). These systems are either integrated $[2,5,6]$ or the biological treatment and electricity generation processes are separated from the filtration process [7]. In integrated AnEMBR systems, electrically conductive, catalytic and porous metal or polymer-based hollow fibers (HF) function simultaneously as both cathodes (for hydrogen evolution) and membranes (for the filtration of the treated water) $[2,5,6,8]$. These dual-function cathodes $[1,9,10]$ have several advantages including the reduction in operation and capital cost, and the footprint required for treatment. Additionally, the small radial dimensions of the HF architecture allows for an increased cathode packing density, a critical aspect for the scale-up of AnEMBR system [2].

Membrane fouling is a considerable challenge in both aerobic and anaerobic membranebased bioreactors and it is more problematic under anaerobic than aerobic conditions [2]. Gas sparging is the most widely used method for membrane fouling control in anaerobic membrane bioreactor (AnMBR). However, this method is energy intensive $\left(0.6-1.6 \mathrm{kWh} / \mathrm{m}^{3}\right)[11]$ and consumes 36\% of the total MBR operating costs [12]. An important advantage of using dualfunction cathodes in AnEMBRs is the inherent biofouling control of the cathodes related to the in-situ production of biogas $\left(\mathrm{H}_{2}, \mathrm{CH}_{4}\right)$ and its scouring effect on the membrane surface $[2,5,6]$. In AnMBRs, HF that are located within the modules might not be accessible by the rising gas bubbles, leading to lower local shear force and higher fouling rates [13, 14], while in AnEMBRs 
membrane fibers are accessible to gas bubbles even at a high packing density since bubbles evolve directly on the fiber surface.

In MECs, applied voltage and specific cathode surface area (SCSA), or the cathode surfacearea-per-reactor volume, play a vital role in the hydrogen production rates in MECs [15], and thus can affect fouling propensity in the AnEMBR system. Katuri et al. [2] observed a significant drop in transmembrane pressure (TMP) when they increased the applied voltage from $0.5 \mathrm{~V}$ to 0.9 V in an AnEMBR due to increased hydrogen production rates. Similarly, Werner et al [6] showed that onset of biofouling was delayed and minimized in AnEMBR operated at $0.9 \mathrm{~V}$, compared to those at $0.7 \mathrm{~V}$ due to higher rates of hydrogen production. To the best of our knowledge, there have been no systematic studies examining the effect of SCSA in AnEMBR. Therefore, the objective of this study was to investigate the effect of different SCSAs on the performance of AnEMBR in terms of organic removal, gas production, energy recovery and membrane fouling. High-speed camera to capture a video stream of the in-situ gas generation on the cathode surface was used for the first time, providing novel insights on the effect of bubble size, distribution and frequency (evolution rates) on controlling membrane fouling.

\section{Materials and Methods}

\subsection{Preparation of dual-function cathodes}

A combined phase-inversion/sintering method was used to fabricate the nickel-based hollow fiber membranes (Ni-HFM) as previously described [2]. The detailed characterization of the NiHFM in terms of pore size distribution, hydraulic permeability and surface roughness is provided in the Supplementary Material and Fig. S1 and S2. The submerged ends of the cylindrical fibers 
were sealed with non-conductive epoxy and the tops of the fibers left open for effluent filtration. The lateral surface area (L) of each fiber was calculated using the equation:

$\mathrm{L}=2 \pi \mathrm{rh}$

where $r$ is the outer radius of the fiber and $h$ is the length of the fiber. The total lateral surface area $\left(\mathrm{m}^{2}\right)$ of the fibers was normalized to the reactor volume $\left(\mathrm{m}^{3}\right)$ to calculate the required fiber numbers for each experimental SCSA variation.

\subsection{Construction and operation}

The single-chamber AnEMBRs (Fig. S3) were constructed using a screw-capped borosilicate glass bottles, with $300 \mathrm{~mL}$ and $280 \mathrm{~mL}$ total and working volumes, respectively [5]. The caps and bottles were modified with appropriate ports to place the anodes, cathodes, gas collection bag and for sampling. Heat-treated $\left(450^{\circ} \mathrm{C}\right.$ for $\left.15 \mathrm{~min}\right)$ graphite brushes containing a titanium core $(4 \mathrm{~cm} \times 2.5 \mathrm{~cm}$, PANEX $33160 \mathrm{~K}$, ZOLTEK) served as anode. The anode and cathode were positioned vertically, approximately $2 \mathrm{~cm}$ apart.

The HFs were bundled into cone-shaped cathodes for AnEMBR I, AnEMBR II and in AnEMBR III to get a SCSA of $2 \mathrm{~m}^{2} / \mathrm{m}^{3}, 4 \mathrm{~m}^{2} / \mathrm{m}^{3}$ and $8 \mathrm{~m}^{2} / \mathrm{m}^{3}$, respectively. Each fiber bundle was held together using insulated copper wire (for current collection) and conductive silver epoxy. An additional layer of non-conductive epoxy was applied around the silver epoxy to prevent the contact of silver with the electrolyte (i.e. feed) during reactor operation. A silicone tube $(34 \mathrm{~cm}$ length; $8 \mathrm{~mm}$ diameter) was used to connect the top of each cathode fiber bundle to a peristaltic pump for filtration. Teflon tape and epoxy were applied wherever necessary on all the connections to ensure a proper seal. 
Each experimental variation was studied in duplicate reactors and the results were reported as average of duplicate readings. The reactors were operated in batch mode with an applied voltage of $0.7 \mathrm{~V}$ using an external power source (3645 A; Circuit Specialists, Inc., AZ). The AnEMBRs were inoculated with anaerobic digester sludge $(10 \% \mathrm{v} / \mathrm{v}$, Manfouha Wastewater Treatment Plant, Riyadh, KSA) and fed with $10 \mathrm{mM}$ acetate for the first three batches, which was then lowered to $6 \mathrm{mM}$ in subsequent batches until the end of the experiment. The feed medium was buffered with bicarbonate, and its composition was as follows (g/L): $\mathrm{NH}_{4} \mathrm{Cl}, 1.5 ; \mathrm{Na}_{2} \mathrm{HPO}_{4}, 0.6$; $\mathrm{KCl}, 0.1$; and 2.5, $\mathrm{Na}_{2} \mathrm{HCO}_{3}$, and trace minerals and vitamin solutions $(\mathrm{pH} 7.0$, conductivity $=$ $6.84 \mathrm{mS} / \mathrm{cm})$ [5]. The media was sparged anaerobically for 1 hour using a $\mathrm{N}_{2}: \mathrm{CO}_{2}(80: 20)$ gas mixture and then autoclaved. A data logger (ADC 24, PicoLog, UK) was used to measure the voltage across an external resistor $\left(\mathrm{R}_{\mathrm{ex}}=10 \Omega\right)$. The drop of voltage to within $10 \%$ of the peak reading signaled the end of each batch for media replacement and sampling. The reactors were operated for a total of 75 days.

\subsection{Membrane chemical cleaning}

The Ni-HFM bundles were removed on Day 56 for cleaning. They were rinsed with a $1 \%$ $\mathrm{H}_{2} \mathrm{SO}_{4}$ solution for 5 to 10 seconds, and then washed with distilled water. The bundles were then sonicated with a sonicator (Branson, M1800, USA) for 10 minutes, dried at $50^{\circ} \mathrm{C}$ for 1 hour, sonicated again for 5 minutes, and finally washed with distilled water before being returned to the reactors. 


\subsection{Measurement and analyses}

Acetate and gas $\left(\mathrm{H}_{2}, \mathrm{~N}_{2}, \mathrm{CH}_{4}\right.$ and $\left.\mathrm{CO}_{2}\right)$ measurements were done at the end of each batch cycle. Details on gas measurements, coulombic efficiency (CE), cathodic hydrogen $\left(\mathrm{Q}_{\mathrm{H} 2}\right)$ and methane ( $\left.\mathrm{Q}_{\mathrm{CH} 4}\right)$ production rates and recovery ( $\mathrm{r}_{\mathrm{Cat}}$ ) used to evaluate the performance of AnEMBRs are provided in Supplementary Material.

The TMP was monitored as an indicator for biofouling of the cathodic membranes. It was recorded for alternating batches from Day 15 till the end of the experiment by applying a flux (J) of $16 \mathrm{~L} / \mathrm{m}^{2} / \mathrm{h}$ (LMH), for all AnEMBRs. The flux, i.e. the flow rate of filtrate passing through the membrane per unit area of the membrane, was obtained using the equation [16]:

$Q_{p}=J \times A_{m}$

where, $\mathrm{J}=$ flux $, \mathrm{L} / \mathrm{m}^{2} / \mathrm{h}, \mathrm{Q}_{\mathrm{p}}=$ filtrate flow rate through membrane, $\mathrm{L} / \mathrm{h}$ and $\mathrm{A}_{\mathrm{m}}=$ surface area of the membrane, $\mathrm{m}^{2}$. The required filtrate flow rate was obtained by adjusting the speed (rpm) of the peristaltic pump (Masterfelx L/S, Cole-Parmer, Vernon Hills, IL). The TMP was measured using a pressure transducer (68075-32, Cole-Parmer Instrument Company) and recorded using a data acquisition system (LabVIEW, National Instruments) connected to a computer.

The bubble generation was captured on Day 35 and Day 55 (8 hours after the batch change) using long-distance microscopes with adjustable magnification (Leica Z16 APO), giving a corresponding pixel resolution between 1.9 and 30.7 microns. The bubble rise was recorded by Phantom v2511 CMOS high-speed video camera (Fig. 1) using the Phantom Camera Control software (Vision Research) at frame rates of 25,000 frames-per-second with 1280 x $800 \mathrm{px}$ resolution. Back-lighting used a $350 \mathrm{~W}$ metal-halide lamp (Sumita), which was shone onto a diffuser [17]. 


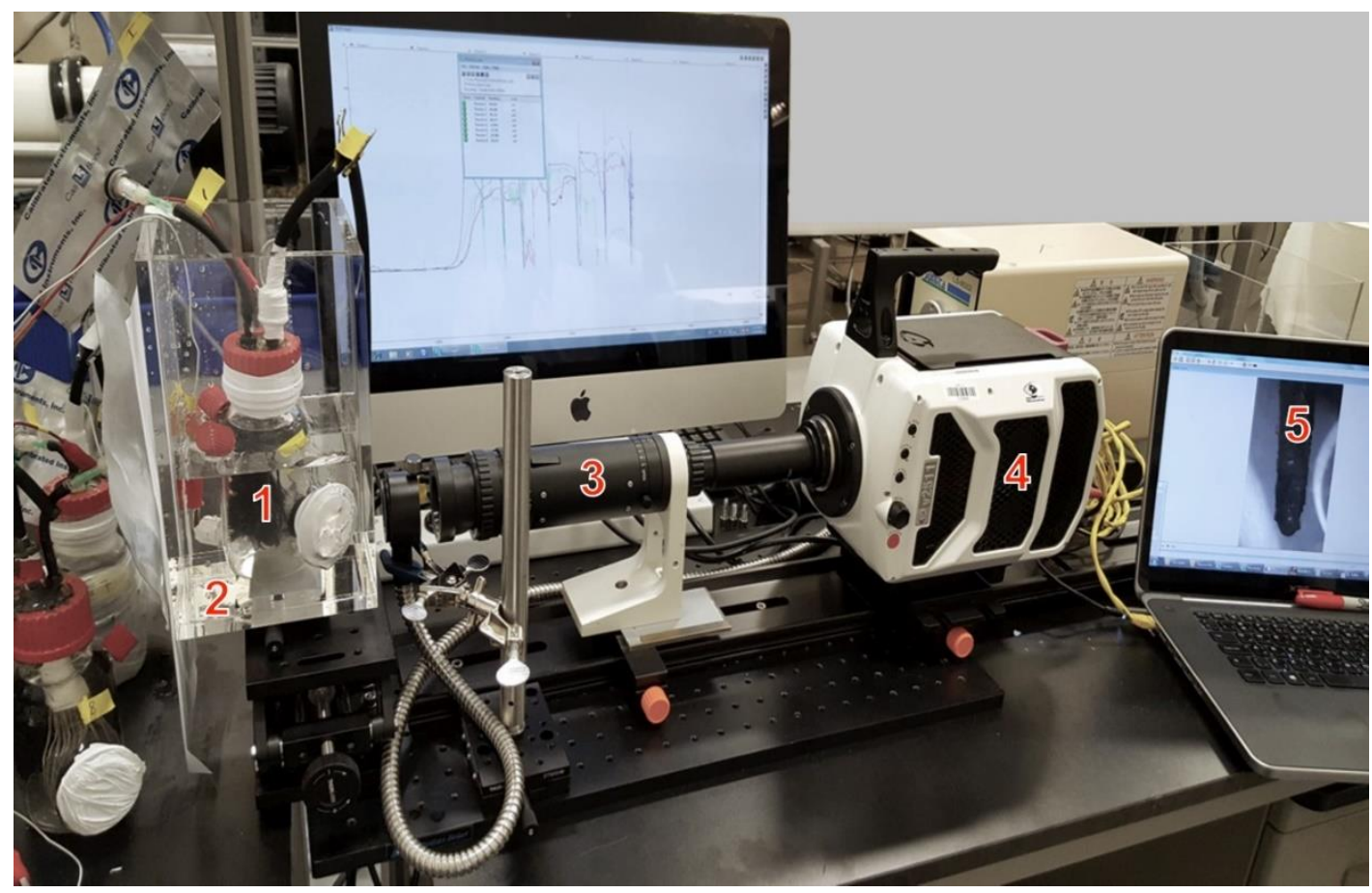

Fig. 1. A combination of high-resolution microscopic and wide-view macroscopic techniques integrating a state-of-the-art high-speed video camera were utilized for visualization of in-situ gas bubble generation. The different components are (1) AnEMBR, (2) the glycerol immersion tank in which the reactors were placed for image capturing, (3) the long-distance microscopic lens attached to (4) the high-speed video camera, and (5) the data acquisition system.

Since the reactors were made of borosilicate glass, this would lead to a visible distortion when light passes through the air/glass and glass/liquid interfaces. Glycerol has a refractive index of 1.47, which almost matches that of borosilicate glass, thus eliminating light distortion at one interface and greatly improving the image quality. Therefore, each reactor was completely submerged into a square acryl glass tank filled with pure glycerol, as shown in Fig. 1.

With the fine pixel resolution of the high-speed video frames we can measure directly the bubble diameters of all bubbles released from the cathode surface over the entire duration of the 
video clip. We use this to quantify the probability density distribution (pdf) of the sizes of the released bubbles. In other words, we can determine the relative release frequency of different bubble sizes. In combination with the volume of each bubble ((4/3) $\left.\pi r^{\wedge} 3\right)$ and the total quantity of biogas produced during the batch operating time of the respective AnEMBR, which is determined using a gas chromatograph (GC, model 310, SRI Instruments), we can estimate the number of pinch-off events per second, in each bubble size-range.

At day 56, and before membrane cleaning, samples of the fibers were taken for scanning electron microscopy (SEM, Quanta 200D, FEI, The Netherlands). Samples were sequentially dehydrated in a series of ethanol solutions (30-100\% ethanol) and oven-dried for 2 hours at $30^{\circ} \mathrm{C}$. Directly before imaging, samples were sputter-coated with gold palladium for $30 \mathrm{~s}$ at 25 $\mathrm{mA}$ current in an argon atmosphere to improve sample conductivity.

At the end of the experiment, a small piece of a single fiber $(\sim 0.5 \mathrm{~cm})$ was cut from each reactor and stored at $-80^{\circ} \mathrm{C}$ for microbial community characterization. Genomic DNA was extracted using the Power Soil DNA extraction kit (MO BIO Laboratories Inc., U.S.A.) as per the manufacturer's instructions. The DNA concentration was measured with Quant-iT BroadRange dsDNA Assay kit (Q33130, Life Technologies, Carlsbad, CA, USA) using the manufacturer's protocol. The 16S rRNA genes were amplified with forward primer Pro341F (5'CCTACGGGNBGCASCAG-3') and reverse primer Pro805R (5'GACTACNVGGGTATCTAATCC-3') [18] targeting the V3-V4 region. The V3-V4 16S rRNA sequencing libraries were prepared by a custom protocol based on Illumina (see Supplementary Material). The purified sequencing libraries were pooled in equimolar concentrations and diluted to $4 \mathrm{nM}$. The samples were paired-end sequenced (2x301bp) on a MiSeq (Illumina, Carlsbad, CA, USA) using a MiSeq Reagent kit v3, 600 cycles (Illumina) following the standard guidelines 
for preparing and loading samples on MiSeq. The reads were dereplicated and formatted for use in the UPARSE workflow [19]. The dereplicated reads were clustered, using the usearch v. 7.0.1090 -cluster_otus command with default settings. Operational taxonomic unit (OTU) abundances were estimated using the usearch v. 7.0.1090 -usearch_global command with 97\% sequence identity threshold. Taxonomy was assigned using the RDP classifier [20] as implemented in the parallel_assign_taxonomy_rdp.py script in QIIME [21], using the MiDAS database v.1.20 [22].

\section{Results and discussion}

\subsection{Effect of SCSA on current density}

Current generation and gas production were observed starting from the second batch onwards in all reactors. For the first two batches (10 mM acetate), current generation dropped within $24 \mathrm{~h}$ in AnEMBR III, but was sustained for up to $36 \mathrm{~h}$ in AnEMBR I and AnEMBR II. By the third batch, all of the AnEMBRs had a consistent batch duration time $(\sim 35 \mathrm{~h})$, at which point the feed was switched to a lower acetate concentration $(6 \mathrm{mM})$ till the end of the experiment.

Figure 2 represents the maximum current density recorded for each batch of the three AnEMBRs. The current density increased gradually in the first 30 days of operation (Fig. 2). The average maximum current density in the first 30 days of operation was slightly higher in AnEMBR III $\left(25.7 \pm 0.001 \mathrm{~A} / \mathrm{m}^{3}\right)$ than AnEMBR I $\left(22.5 \pm 0.002 \mathrm{~A} / \mathrm{m}^{3}\right)$ and II $\left(23.1 \pm 0.003 \mathrm{~A} / \mathrm{m}^{3}\right)$. 

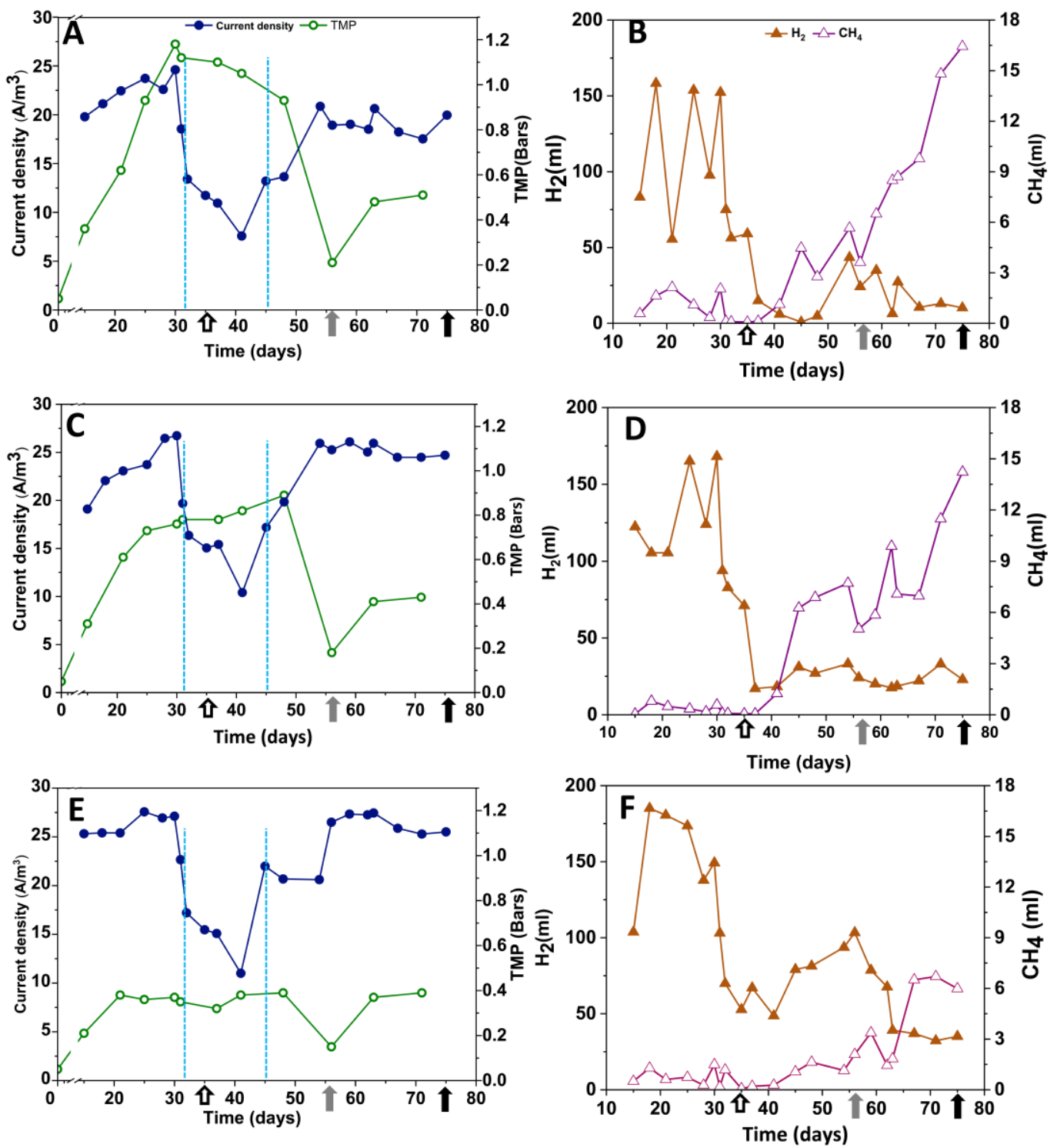

Fig. 2. Peak volumetric current densities, TMP, and volume of gas for AnEMBRs with (A and B) $2 \mathrm{~m}^{2} / \mathrm{m}^{3}$ (C and D) $4 \mathrm{~m}^{2} / \mathrm{m}^{3}$ and (E and F) $8 \mathrm{~m}^{2} / \mathrm{m}^{3}$ SCSA. Values represent averages of duplicate reactors. Each data point in the plot represents the respective performance of individual batch cycles. Unfilled arrows represent day 35 when the high-speed camera was used to capture the in-situ bubble generation, grey arrows represent day 56 when the Ni-HFMs were sampled for SEM imaging followed by physical/ chemical cleaning, and the black arrows represent day 75 when biomass samples were collected for microbial community analysis. The dashed vertical lines cover the region where unintentional disturbance in $\mathrm{pH}$ was induced. 
A significant decrease in current density was observed in all the reactors on day 31 . This was possibly due to a change in media preparation, where pure $\mathrm{N}_{2}$ gas was used to anaerobically sparge the media instead of the $\mathrm{N}_{2}: \mathrm{CO}_{2}$ gas mixture. This led to an increase in media $\mathrm{pH}(>9)$ that negatively affected the electrochemical activity of the biofilm $[23,24]$. Once the $\mathrm{pH}$ disturbance was resolved on day 45 , reactor performance was restored with stable current generation within few batches of operation, demonstrating the robustness of the AnEMBR biofilms to $\mathrm{pH}$ disturbance.

To evaluate the effect of chemical cleaning on the electrocatalytic performance of the NiHFM, the cathode bundles from the three AnEMBRs were chemically cleaned on day 56 (represented by the black arrow in Fig. 2). Irrespective of experimental variations, the performance of all AnEMBRs remained stable after the cathode cleaning which correlated with earlier reports [2], and supports the assumed structural and chemical integrity of Ni-HFM.

\subsection{Effect of SCSA on acetate removal and CE}

Acetate removal did not appear to be significantly affected by the SCSA during normal operation, although AnEMBR III displayed higher removal during the $\mathrm{pH}$ disturbance period compared to the other reactors (Table 1). Irrespective of SCSA, >100\% CEs were calculated for the first 30 days of operation (Table 1). The highest CEs were observed at Day 15, with AnEMBR III having a CE of $119.20 \pm 0.18 \%$, followed by AnEMBR II and AnEMBR I at $113.60 \pm 0.31 \%$ and $110.30 \pm 0.22 \%$ respectively. Coulombic efficiencies $>100 \%$ have been reported previously in AnEMBR due to hydrogen recycling by exoelectrogens using hydrogen as the electron donor [5, 6]. Also, the dominance of Acetobacterium at the cathode (see section 3.6) 
suggests the possibility of acetate production, which can then be utilized at the anode resulting in $\mathrm{CE}>100 \%$. With the single-chamber design used in the current study it was not possible to determine the contribution of acetate generation from the cathode. The AnEMBRs showed low CE during days 35- 45 due to the associated issues with the feed preparation, as previously discussed. During this period, the $\mathrm{r}_{\mathrm{Cat}} \mathrm{H}_{2}$ was the highest (Table 1), which further confirms the possibility of $\mathrm{H}_{2}$ utilization at the anode resulting in increased CE. As with the current density, the $\mathrm{CE}$ increased after resolving the $\mathrm{pH}$ issue. 
1 Table 1

2 Performance of the different AnEMBRs operated with varying specific cathode surface area (SCSA).

\begin{tabular}{|c|c|c|c|c|c|c|}
\hline $\begin{array}{l}\text { Time } \\
\text { (days) }\end{array}$ & $\begin{array}{c}\text { Acetate removal } \\
(\%)\end{array}$ & $\mathrm{CE}(\%)$ & $\mathrm{Q}_{\mathrm{H} 2}\left(\mathrm{~m}^{3} / \mathrm{m}^{3} / \mathrm{d}\right)$ & $\mathrm{Q}_{\mathrm{CH} 4}\left(\mathrm{~m}^{3} / \mathrm{m}^{3} / \mathrm{d}\right)$ & $\mathrm{r}_{\text {Cat }} \mathrm{H}_{2}(\%)$ & $\mathrm{r}_{\mathrm{Cat}} \mathrm{CH}_{4}(\%)$ \\
\hline \multicolumn{7}{|c|}{$2 \mathrm{~m}^{2} / \mathrm{m}^{3}($ AnEMBR I) } \\
\hline 15 & $91.76 \pm 0.25$ & $110.30 \pm 0.22$ & $0.31 \pm 0.02$ & $0.0012 \pm 0.0005$ & $55.62 \pm 5.13$ & $0.32 \pm 0.24$ \\
\hline 27 & $90.88 \pm 0.19$ & $98.00 \pm 0.42$ & $0.25 \pm 0.14$ & $0.0009 \pm 0.0004$ & $63.01 \pm 3.46$ & $0.91 \pm 0.91$ \\
\hline 37 & $77.23 \pm 0.85$ & $36.60 \pm 0.63$ & $0.03 \pm 0.08$ & $0.0003 \pm 0.0001$ & $25.71 \pm 1.87$ & $0.87 \pm 0.31$ \\
\hline 57 & $89.56 \pm 0.01$ & $104.70 \pm 0.18$ & $0.10 \pm 0.03$ & $0.0066 \pm 0.0002$ & $27.71 \pm 7.25$ & $8.74 \pm 0.14$ \\
\hline 75 & $89.17 \pm 0.15$ & $85.50 \pm 0.17$ & $0.02 \pm 0.05$ & $0.0466 \pm 0.0100$ & $7.81 \pm 1.17$ & $28.88 \pm 0.74$ \\
\hline \multicolumn{7}{|c|}{$4 \mathrm{~m}^{2} / \mathrm{m}^{3}$ (AnEMBR II) } \\
\hline 15 & $90.64 \pm 1.28$ & $113.60 \pm 0.31$ & $0.36 \pm 0.01$ & $0.0010 \pm 0.0004$ & $70.00 \pm 3.86$ & $0.41 \pm 0.18$ \\
\hline 27 & $89.49 \pm 2.25$ & $103.00 \pm 0.26$ & $0.29 \pm 0.02$ & $0.0005 \pm 0.0002$ & $68.80 \pm 5.37$ & $0.44 \pm 0.04$ \\
\hline 37 & $78.92 \pm 2.27$ & $52.10 \pm 0.13$ & $0.20 \pm 0.11$ & $0.0002 \pm 0.0001$ & $122.19 \pm 5.07$ & $0.55 \pm 0.06$ \\
\hline 57 & $90.85 \pm 0.11$ & $115.90 \pm 0.18$ & $0.08 \pm 0.02$ & $0.0067 \pm 0.0022$ & $20.93 \pm 3.85$ & $6.87 \pm 0.05$ \\
\hline 75 & $89.46 \pm 0.09$ & $95.30 \pm 0.13$ & $0.06 \pm 0.02$ & $0.0280 \pm 0.0100$ & $15.47 \pm 4.42$ & $19.5 \pm 0.60$ \\
\hline \multicolumn{7}{|c|}{$8 \mathrm{~m}^{2} / \mathrm{m}^{3}$ (AnEMBR III) } \\
\hline 15 & $92.91 \pm 1.48$ & $119.20 \pm 0.18$ & $0.42 \pm 0.03$ & $0.0009 \pm 0.0002$ & $77.00 \pm 3.05$ & $0.82 \pm 0.26$ \\
\hline 27 & $93.64 \pm 2.25$ & $107.4 \pm 0.09$ & $0.35 \pm 0.03$ & $0.0007 \pm 0.0004$ & $81.01 \pm 4.95$ & $0.63 \pm 0.86$ \\
\hline 37 & $82.69 \pm 1.95$ & $54.1 \pm 0.26$ & $0.23 \pm 0.01$ & $0.0004 \pm 0.0006$ & $133.15 \pm 8.85$ & $0.95 \pm 0.13$ \\
\hline 57 & $93.46 \pm 0.46$ & $126.8 \pm 0.36$ & $0.24 \pm 0.16$ & $0.0049 \pm 0.0016$ & $51.47 \pm 2.26$ & $4.16 \pm 0.96$ \\
\hline 75 & $93.76 \pm 0.63$ & $97.2 \pm 0.18$ & $0.11 \pm 0.03$ & $0.0175 \pm 0.0088$ & $24.00 \pm 2.29$ & $16.90 \pm 1.02$ \\
\hline
\end{tabular}




\subsection{Effect of SCSA on biogas production}

Hydrogen gas was the predominant component of the biogas, while methane production reached detectable limits from day 48 onwards for all reactors (Fig. S4). Overall, $\mathrm{r}_{\mathrm{Cat}} \mathrm{H}_{2}$ and $\mathrm{Q}_{\mathrm{H} 2}$ was proportional to the SCSA (i.e., higher $\mathrm{Q}_{\mathrm{H} 2}$ and $\mathrm{r}_{\mathrm{Cat}} \mathrm{H}_{2}$ was observed for $8 \mathrm{~m}^{2} / \mathrm{m}^{3}$ compared to $2 \mathrm{~m}^{2} / \mathrm{m}^{3}$ and $4 \mathrm{~m}^{2} / \mathrm{m}^{3}$ ), while that of methane was inversely proportional (Table 1 ). The maximum Qн2 was observed in AnEMBR III on day 15 with $0.42 \pm 0.03 \mathrm{~m}^{3} / \mathrm{m}^{3} / \mathrm{d}$, followed by $0.36 \pm 0.01$ and $0.31 \pm 0.01 \mathrm{~m}^{3} / \mathrm{m}^{3} / \mathrm{d}$ for AnEMBR II and AnEMBR I, respectively (Table 1). The Q ${ }_{\mathrm{H} 2}$ and $\mathrm{r}_{\mathrm{Cat}} \mathrm{H}_{2}$ decreased with time in all the reactors, though it was a more pronounced decrease in AnEMBR I than in AnEMBR III (Table 1). On the other hand, the maximum methane production rate $\left(\mathrm{Q}_{\mathrm{CH} 4}\right)$ and cathodic recovery of methane $\left(\mathrm{r}_{\mathrm{Cat}} \mathrm{CH}_{4}\right)$ increased with time in all the AnEMBRs (Table 1), with higher $\mathrm{Q}_{\mathrm{CH} 4}$ and $\mathrm{r}_{\mathrm{Cat}} \mathrm{CH}_{4}$ observed in AnEMBR I than AnEMBR III. The fact that the total $\mathrm{rCat}\left(\mathrm{H}_{2}+\mathrm{CH}_{4}\right)$ was lower than $100 \%$ at most of the time suggests the possibility of other sinks of electron such as $\mathrm{H}_{2}$ scavengers (exoelectrogens at the anode and Acetobacterium at the cathode).

\subsection{Bubble flow rate and spatial distribution}

The in-situ evolution, size distribution and frequency $(\mathrm{Hz})$ of gas bubbles was analyzed by direct imaging with a high-speed video camera on day 35 and day 55 of operation, providing novel insights on their effect on biofouling control in the AnEMBR system. The frequency of individual bubbles was derived from the high-speed image captures (bubble size and probability density function) (Table S1) and GC analysis (quantity of gas). The bubble sizes ranged from 45 $\mu \mathrm{m}$ to $213 \mu \mathrm{m}$ (Table S1) with a different bubble distribution in the three AnEMBRs (Fig. 3) with more smaller bubbles (45-114 $\mu \mathrm{m}$ ) in AnEMBR III than AnEMBR I and II (Fig. 3). 

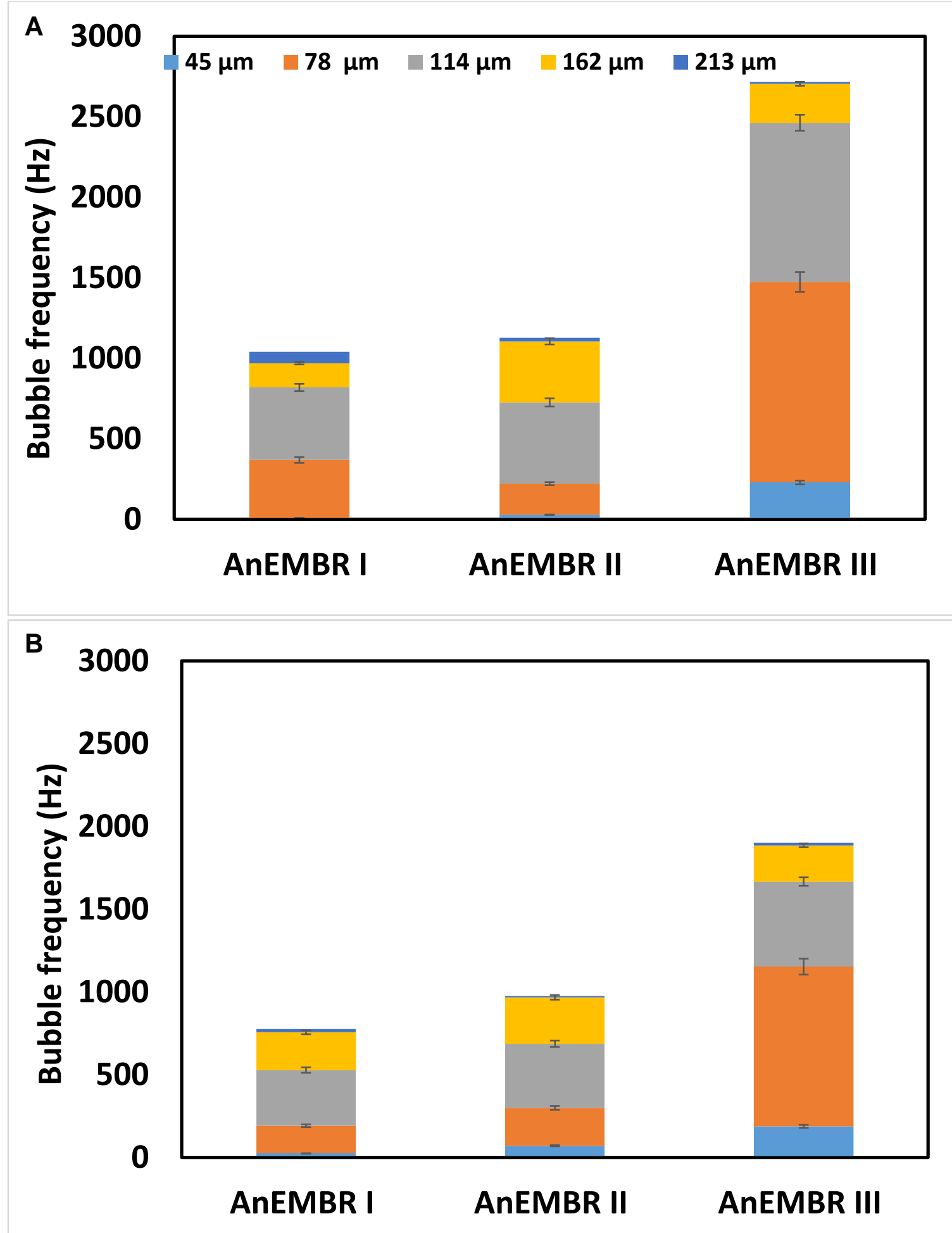

Fig. 3. The accumulated frequency of all gas bubbles on the cathode surface of the AnEMBRs on day 35 (A) and day 55 (B). 
The frequencies of the smaller-sized bubbles $(45 \mu \mathrm{m}$ and $114 \mu \mathrm{m})$ decreased with decreasing SCSA (Fig. 3). The main difference between the two periods of measurements (i.e., day 35 and day 55) was in bubble frequency where lower bubble frequency was observed on day 55 (Fig. 3b) compared to day 35 (Fig. 3a). By the time the second measurement was conducted, hydrogenotrophic methanogenesis was already established in all the reactors. This altered the bubble frequency distribution (Fig. 3b) on the cathode surface due to the conversion of $\mathrm{H}_{2}$ to $\mathrm{CH}_{4}$ (four moles of $\mathrm{H}_{2}$ is consumed to produce one mole of $\mathrm{CH}_{4}$ ) by hydrogenotrophic methanogens. Nevertheless, the distribution of gas bubble size and the trend in bubble frequency as a function of SCSA was similar between the two periods. Overall, increased biogas quantity and small bubble distribution led to the highest frequency, or rate of bubble formation, in AnEMBR III.

The captured videos revealed that the AnEMBRs not only displayed variations in bubble sizes and frequency but also in nucleation (initiation) sites on the cathode surface (Supplementary videos). Liquid phase supersaturation due to gas diffusion is a precondition for bubble nucleation $[25,26]$. Single-phase free convection causes nucleation at certain active sites on the electrode surface [27]. There are several different types of bubble nucleation, which is influenced by a number of factors in an electrolysis cell [28, 29]. Of importance to the results reported here are Type II and III nucleation (bubbles nucleate from new sites), and Type IV (initial bubble provides an opportunity for a next bubble to form, grow and detach at the same site) [28]. AnEMBR I showed Type II and III nucleation, whereas AnEMBR II and III displayed Type IV nucleation with bubbles evolving from the bottom of the cathode fibers. It is beyond the scope of this study to explain specific reasons for why the nucleation sites varied with SCSA. 
However, the type of nucleation may have been affected by the amount of biogas produced on the cathode surface, since the energy barrier is much higher for Type II and III compared to Type IV nucleation [28].

\subsection{Membrane biofouling}

Throughout the experiment, the AnEMBR with $8 \mathrm{~m}^{2} / \mathrm{m}^{3}$ showed distinctly low TMP compared to the AnEMBRs with $2 \mathrm{~m}^{2} / \mathrm{m}^{3}$ and $4 \mathrm{~m}^{2} / \mathrm{m}^{3}$. These variations appear to be due to the cumulative impact of bubble size, distribution and nucleation sites (discussed in previous section), and rates of hydrogen production rate (i.e., $\mathrm{Q}_{\mathrm{H} 2}$ ) (Table 1). The magnitude of changes in the rates of hydrogen production (i.e., $\mathrm{Q}_{\mathrm{H} 2}$ ) (Table 1) influenced the TMP in the respective AnEMBRs (Fig. 2), with TMP being inversely proportional to $\mathrm{Q}_{\text {н2. }}$ Previous studies on AnEMBR $[2,6]$ also revealed reduced TMP with increased hydrogen production rates. During the $\mathrm{pH}$ disturbance period (i.e., day 31 to 45), the TMP in AnEMBR I decreased by 27\% (1.18 to 0.93 bars). In contrast the TMP in AnEMBR II increased by $12 \%$ ( 0.78 to 0.89 bars). This difference in behavior in TMP between the two reactors could be due to differences in biofilm thickness. The in-situ gas $\left(\mathrm{H}_{2} / \mathrm{CH}_{4}\right)$ evolution on the cathode surface can cause detachment of the biofilm. This detachment due to the in-situ HER reaction is expected to be higher for thicker biofilms. The TMP in AnEMBR I reached 1.18 bars on day 30 compared to 0.8 bars for AnEMBR II. This suggests that the propensity of biofouling in AnEMBR I was higher than AnEMBR II. This was also supported by SEM images (Fig. 5) taken on day 56, where the fouling layer formed on the cathode of AnEMBR I was thicker (visually) than AnEMBR II. However, scouring of biofilm due to the in-situ HER reaction is more effective when the biofilm layer is thicker $[2,6]$. For example, increasing the applied voltage from $0.7 \mathrm{~V}$ to $0.9 \mathrm{~V}$ was not 
effective in reducing TMP on Ni-HFM with biofilm thickness of $0.4-0.5 \mu \mathrm{m}$ [6]. In contrast, increasing the applied voltage from $0.5 \mathrm{~V}$ to $0.9 \mathrm{~V}$ significantly reduced TMP on Ni-HFM with biofilm thickness of $4 \mu \mathrm{m}[2]$.

The relationship of TMP to $\mathrm{Q}_{\mathrm{H} 2}$ variations can be a result of i) faster hydrogen gas bubble evolution compared to methane gas bubbles as hydrogen requires less time for molecular diffusion [30], ii) hydrogen gas bubbles travel at a faster velocity than methane due to the lower density of hydrogen $\left(0.0898 \mathrm{~kg} / \mathrm{m}^{3}\right)$ compared to methane $\left(0.656 \mathrm{~kg} / \mathrm{m}^{3}\right)$ gas, and iii) the volume of gas increases with hydrogen production compared to methane since one mole of acetate generates four moles of hydrogen but only one mole of methane.

As previously mentioned, variations in bubble frequency and size distribution were observed (Fig. 3) which could also strongly influence the hydrodynamics of the foulant layer of the membrane $[31,32]$. AnEMBR III showed more bubble frequency than AnEMBR I and II that was well correlated with TMP of the respective bioreactor (Fig. 2 and 3). AnEMBR I showed a 0.006 bubble distribution at $45 \mu \mathrm{m}$, whereas AnEMBR II and AnEMBR III showed 0.025 and 0.084 bubble distribution (Table S1). The shape of the bubbles is controlled by their size [32, 33], which in turn could influence the scouring efficiency of the bubbles. Smaller bubbles are more capable of foulant removal [34]. Also, bubble slip velocity, which is the velocity of the gas phase relative to that of the liquid phase, is influenced by the size of the bubble. The slip velocity decreases with increasing bubble size and increases with decreasing bubble size [35, 36], which in turn affects the control of membrane fouling. Therefore, a unit of gas that has a high frequency of small bubbles than large bubbles creates more bubble slip velocity and shear force on the liquid and gas interface. This means that at a given sparging flow rate, smaller bubbles increase the number of shear events compared to larger bubbles [37]. Additionally, higher number of 
bubbles increases bubble velocity $[38,39]$, resulting in stronger scouring within the bubbles' zone of influence on the membrane. Taken together, the cumulative effect of widespread smaller bubbles with higher frequency appeared to limit fouling layer formation on the cathode surface for AnEMBR III (Fig. 3) in comparison to the other two AnEMBRs.

Furthermore, the evolution of gas bubbles from the bottom of the fibers using Type IV nucleation (AnEMBRs II and III) appeared to be advantageous to control fouling. This may be because the detachment of the gas bubbles from the bottom of the electrode surface caused more turbulence in the electrode boundary (polarization) layer, which helped in biofoulant removal from the electrode surface.

SEM images of the Ni-HFM sampled on day 56 prior to the chemical cleaning of the membranes showed clear differences in foulant layer morphology among the three AnEMBRs, supporting the TMP results. In AnEMBR I, the fibers' surface was completely covered and the foulant layers formed a cake-like structure, presumably due to EPS matter in the microbial biofilm (Fig. 4B). There were no cake-like structures observed on the fibers in AnEMBR II, however there were more microbial aggregates observed on the fibers (Fig. 4C) and few microbial aggregates observed on Ni-HFM fibers of AnEMBR III. No open pores were visible in AnEMBR I (Fig. 4B), few open or partially closed pores observed in AnEMBR II (Fig. 4C) whereas more clearly open pores observed in AnEMBR III (Fig. 4D). These variations are probably due to the differences in the in-situ bubble scouring in the respective AnEMBRs. The SEM image results correlated with the TMP of respective AnEMBRs (Fig. 2). 

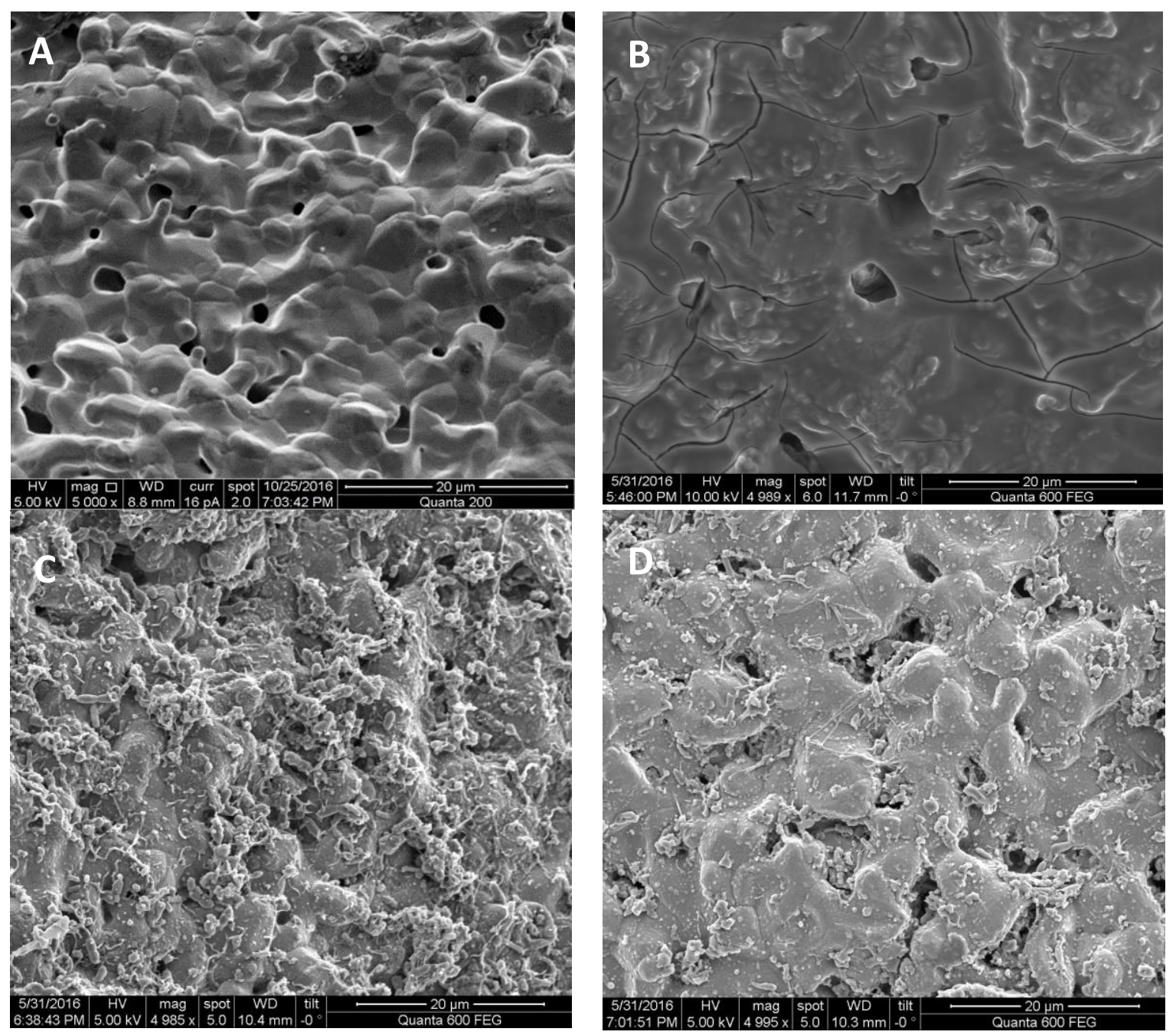

Fig. 4. Scanning electron micrograph images of (A) virgin Ni-HFM and biofouled Ni-HFM at day 56 for (B) $2 \mathrm{~m}^{2} / \mathrm{m}^{3}$ (C) $4 \mathrm{~m}^{2} / \mathrm{m}^{3}$ and (D) $8 \mathrm{~m}^{2} / \mathrm{m}^{3}$.

\subsection{Microbial community composition at the cathode}

Amplicon sequencing results revealed that methanogenic communities were present on the cathode of all AnEMBRs (Fig. 5). The hydrogenotrophic methanogen, Methanocorpusclum, was the dominant methanogen detected on the biofouled Ni-HFM. Methanocorpusculum were present in relatively higher abundance in AnEMBR I (2.5\%), than in AnEMBR II (1.8\%) and AnEMBR III (1.7\%). The relatively high abundance of Methanocorpusculum in AnEMBR I and II correlates with their higher methane generation compared to AnEMBR III (Table 1). The 
dominant bacterial phyla detected on the cathode belonged to Firmicutes and Bacteroidetes, with Acetobacterium (phylum Firmicutes) being the dominant genera ( 52\% of the total sequence reads) detected on the cathode, with the highest relative abundance (62\%) detected in AnEMBR III. Members of the genus Acetobacterium are homoacetogens capable of generating acetate from $\mathrm{CO}_{2}$ and $\mathrm{H}_{2}[40,41]$.

\section{Conclusions}

The results clearly demonstrated the effect of SCSA on the performance of AnEMBR in terms of current density, biogas production and composition, and biofouling rates. The rates of hydrogen production influenced the TMP, with TMP being inversely proportional to $\mathrm{Q}_{\mathrm{H} 2}$. The high frequency of smaller bubbles $(45-114 \mu \mathrm{m})$ and type IV nucleation appeared to limit biofouling on the cathode surface for AnEMBR III, which was operated for 55 days without replacement or cleaning (e.g. backwashing or chemical treatment). The cumulative effect of the above-mentioned factors, along with the variation of microbial community, controlled biofouling and enhanced energy recovery in AnEMBR III operated at SCSA of $8 \mathrm{~m}^{2} / \mathrm{m}^{3}$. The insights provided by this study will be helpful in the up-scaling of AnEMBR for wastewater treatment and reuse and energy recovery. 


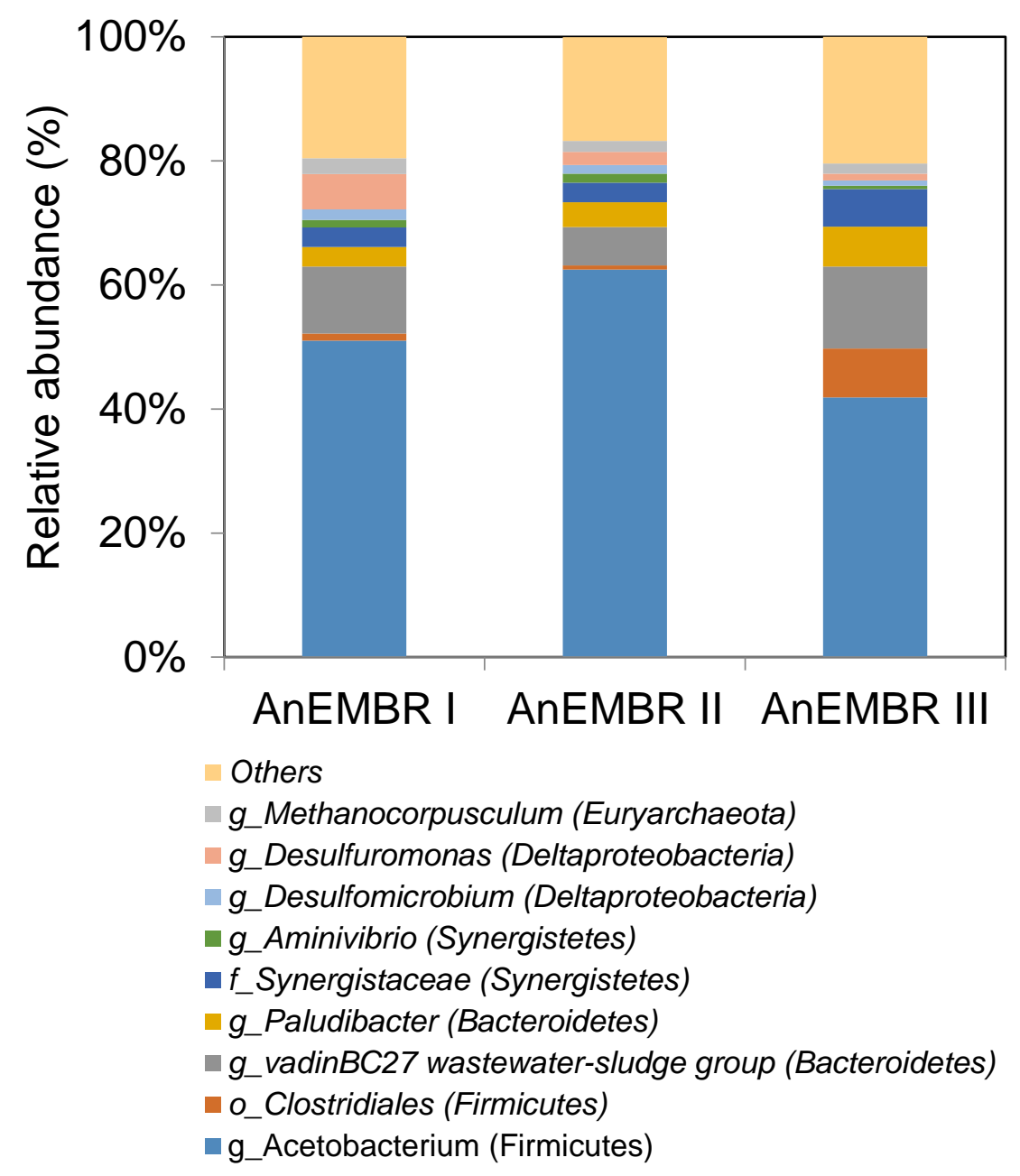

Fig. 5. The average relative abundances (\%) of dominant genera ( $~ 80 \%$ of sequence reads) detected on the cathode of the three AnEMBRs. Others ( 20\% of sequence reads) represent genera with relative abundance < $1 \%$. g: genus; f: family; o: order.

\section{Acknowledgments}

This work was supported by Center Competitive Funding Program (FCC/1/1971-33-01) to P.E.S. from King Abdullah University of Science and Technology (KAUST).

\section{Appendix A. Supplementary data}

Supplementary data associated with this article can be found in the online version. 


\section{References}

[1] K.P. Katuri, S. Kalathil, A. Ragab, B. Bian, M.F. Alqahtani, D. Pant, P.E. Saikaly, Dual-Function Electrocatalytic and Macroporous Hollow-Fiber Cathode for Converting Waste Streams to Valuable Resources Using Microbial Electrochemical Systems, Adv Mater, 30 (2018).

[2] K.P. Katuri, C.M. Werner, R.J. Jimenez-Sandoval, W. Chen, S. Jeon, B.E. Logan, Z.P. Lai, G.L. Arny, P.E. Saikaly, A Novel Anaerobic Electrochemical Membrane Bioreactor (AnEMBR) with Conductive Hollowfiber Membrane for Treatment of Low-Organic Strength Solutions, Environ Sci Technol, 48 (2014) 1283312841.

[3] A.R. Hari, K. Venkidusamy, K.P. Katuri, S. Bagchi, P.E. Saikaly, Temporal Microbial Community Dynamics in Microbial Electrolysis Cells - Influence of Acetate and Propionate Concentration, Front Microbiol, 8 (2017).

[4] L. Lu, Z.Y.J. Ren, Microbial electrolysis cells for waste biorefinery: A state of the art review, Bioresource Technol, 215 (2016) 254-264.

[5] K.P. Katuri, N.M.S. Bettahalli, X.B. Wang, G. Matar, S. Chisca, S.P. Nunes, P.E. Saikaly, A Microfiltration Polymer-Based Hollow-Fiber Cathode as a Promising Advanced Material for Simultaneous Recovery of Energy and Water, Adv Mater, 28 (2016) 9504-+.

[6] C.M. Werner, K.P. Katuri, A.R. Hari, W. Chen, Z.P. Lai, B.E. Logan, G.L. Amy, P.E. Saikaly, GrapheneCoated Hollow Fiber Membrane as the Cathode in Anaerobic Electrochemical Membrane Bioreactors Effect of Configuration and Applied Voltage on Performance and Membrane Fouling, Environ Sci Technol, 50 (2016) 4439-4447.

[7] A.Q. Ding, Q. Fan, R. Cheng, G.D. Sun, M.J. Zhang, D.L. Wu, Impacts of applied voltage on microbial electrolysis cell-anaerobic membrane bioreactor (MEC-AnMBR) and its membrane fouling mitigation mechanism, Chem Eng J, 333 (2018) 630-635.

[8] C. Cuevas, D. Kim, K.P. Katuri, P. Saikaly, S.P. Nunes, Electrochemically active polymeric hollow fibers based on poly(ether-b-amide)/carbon nanotubes, J Membrane Sci, 545 (2018) 323-328.

[9] M.F. Alqahtani, K.P. Katuri, S. Bajracharya, Y. Yu, Z. Lai, P.E. Saikaly, Porous Hollow Fiber Nickel Electrodes for Effective Supply and Reduction of Carbon Dioxide to Methane through Microbial Electrosynthesis, Advanced Functional Materials, 28 (2018) 1804860.

[10] B. Bian, M.F. Alqahtani, K.P. Katuri, D. Liu, S. Bajracharya, Z. Lai, K. Rabaey, P.E. Saikaly, Porous nickel hollow fiber cathodes coated with CNTs for efficient microbial electrosynthesis of acetate from $\mathrm{CO} 2$ using Sporomusa ovata, Journal of Materials Chemistry A, 6 (2018) 17201-17211.

[11] I. Martin, M. Pidou, A. Soares, S. Judd, B. Jefferson, Modelling the energy demands of aerobic and anaerobic membrane bioreactors for wastewater treatment, Environ Technol, 32 (2011) 921-932.

[12] B. Verrecht, T. Maere, I. Nopens, C. Brepols, S. Judd, The cost of a large-scale hollow fibre MBR, Water Res, 44 (2010) 5274-5283.

[13] J. Gunther, D. Hobbs, C. Albasi, C. Lafforgue, A. Cockx, P. Schmitz, Modeling the effect of packing density on filtration performances in hollow fiber microfiltration module: A spatial study of cake growth, J Membrane Sci, 389 (2012) 126-136.

[14] J. Gunther, P. Schmitz, C. Albasi, C. Lafforgue, A numerical approach to study the impact of packing density on fluid flow distribution in hollow fiber module, J Membrane Sci, 348 (2010) 277-286.

[15] D.F. Call, M.D. Merrill, B.E. Logan, High Surface Area Stainless Steel Brushes as Cathodes in Microbial Electrolysis Cells, Environ Sci Technol, 43 (2009) 2179-2183.

[16] U.S. EPA, Membrane Filtration Guidance Manual (2005). 
[17] E.Q. Li, S.A. Al-Otaibi, I.U. Vakarelski, S.T. Thoroddsen, Satellite formation during bubble transition through an interface between immiscible liquids, J Fluid Mech, 744 (2014).

[18] S. Takahashi, J. Tomita, K. Nishioka, T. Hisada, M. Nishijima, Development of a Prokaryotic Universal Primer for Simultaneous Analysis of Bacteria and Archaea Using Next-Generation Sequencing, Plos One, 9 (2014).

[19] R.C. Edgar, UPARSE: highly accurate OTU sequences from microbial amplicon reads, Nat Methods, 10 (2013) 996-+.

[20] Q. Wang, G.M. Garrity, J.M. Tiedje, J.R. Cole, Naive Bayesian classifier for rapid assignment of rRNA sequences into the new bacterial taxonomy, Appl Environ Microb, 73 (2007) 5261-5267.

[21] J.G. Caporaso, J. Kuczynski, J. Stombaugh, K. Bittinger, F.D. Bushman, E.K. Costello, N. Fierer, A.G. Pena, J.K. Goodrich, J.I. Gordon, G.A. Huttley, S.T. Kelley, D. Knights, J.E. Koenig, R.E. Ley, C.A. Lozupone, D. McDonald, B.D. Muegge, M. Pirrung, J. Reeder, J.R. Sevinsky, P.J. Tumbaugh, W.A. Walters, J. Widmann, T. Yatsunenko, J. Zaneveld, R. Knight, QIIME allows analysis of high-throughput community sequencing data, Nat Methods, 7 (2010) 335-336.

[22] S.J. Mcllroy, A.M. Saunders, M. Albertsen, M. Nierychlo, B. Mcllroy, A.A. Hansen, S.M. Karst, J.L. Nielsen, P.H. Nielsen, MiDAS: the field guide to the microbes of activated sludge, Database-Oxford, (2015). [23] S.A. Patil, F. Harnisch, C. Koch, T. Hubschmann, I. Fetzer, A.A. Carmona-Martinez, S. Muller, U. Schroder, Electroactive mixed culture derived biofilms in microbial bioelectrochemical systems: The role of $\mathrm{pH}$ on biofilm formation, performance and composition, Bioresource Technol, 102 (2011) 9683-9690.

[24] K.P. Katuri, P. Kavanagh, S. Rengaraj, D. Leech, Geobacter sulfurreducens biofilms developed under different growth conditions on glassy carbon electrodes: insights using cyclic voltammetry, Chem Commun, 46 (2010) 4758-4760.

[25] H. Matsushima, D. Kiuchi, Y. Fukunaka, K. Kuribayashi, Single bubble growth during water electrolysis under microgravity, Electrochem Commun, 11 (2009) 1721-1723.

[26] S. Lubetkin, The motion of electrolytic gas bubbles near electrodes, Electrochim Acta, 48 (2002) 357375.

[27] H. Vogt, The Role of Single-Phase Free-Convection in Mass-Transfer at Gas Evolving Electrodes .1. Theoretical, Electrochim Acta, 38 (1993) 1421-1426.

[28] S.F. Jones, G.M. Evans, K.P. Galvin, Bubble nucleation from gas cavities - a review, Adv Colloid Interfac, 80 (1999) 27-50.

[29] Y.G. Chirkov, A.G. Pshenichnikov, An Estimate of the Probability of Spontaneous Gas-Bubble Nucleation in Supersaturated Solutions, Sov Electrochem+, 21 (1985) 114-117.

[30] E.C. Delara, R. Kahn, Diffusivity of Hydrogen and Methane Molecules in a-Zeolites - NeutronScattering Measurements and Comparison, Zeolites, 12 (1992) 256-260.

[31] L. Ji, J.T. Zhou, Influence of aeration on microbial polymers and membrane fouling in submerged membrane bioreactors, J Membrane Sci, 276 (2006) 168-177.

[32] Z.F. Cui, S. Chang, A.G. Fane, The use of gas bubbling to enhance membrane processes, J Membrane Sci, 221 (2003) 1-35.

[33] T. Miyahara, K. Tsuchiya, L.S. Fan, Wake Properties of a Single Gas Bubble in a 3-Dimensional Liquid Solid Fluidized-Bed, Int J Multiphas Flow, 14 (1988) 749-763.

[34] M. Takahashi, K. Chiba, P. Li, Formation of hydroxyl radicals by collapsing ozone microbubbles under strongly acidic conditions, J Phys Chem B, 111 (2007) 11443-11446.

[35] M.J. Nieves-Remacha, A.A. Kulkarni, K.F. Jensen, Gas-Liquid Flow and Mass Transfer in an AdvancedFlow Reactor, Ind Eng Chem Res, 52 (2013) 8996-9010.

[36] J. Katz, C. Meneveau, Wake-induced relative motion of bubbles rising in line, Int J Multiphas Flow, 22 (1996) 239-258.

[37] S.R. Smith, Z.F. Cui, R.W. Field, Upper- and lower-bound estimates of flux for gas-sparged ultrafiltration with hollow fiber membranes, Ind Eng Chem Res, 44 (2005) 7684-7695. 
[38] R. Krishna, M.I. Urseanu, J.M. van Baten, J. Ellenberger, Rise velocity of a swarm of large gas bubbles in liquids, Chem Eng Sci, 54 (1999) 171-183.

[39] I. Komasawa, T. Otake, M. Kamojima, Wake Behavior and Its Effect on Interaction between SphericalCap Bubbles, J Chem Eng Jpn, 13 (1980) 103-109.

[40] W.E. Balch, S. Schoberth, R.S. Tanner, R.S. Wolfe, Acetobacterium, a New Genus of HydrogenOxidizing, Carbon Dioxide-Reducing, Anaerobic Bacteria, Int J Syst Bacteriol, 27 (1977) 355-361.

[41] R. Takors, M. Kopf, J. Mampel, W. Bluemke, B. Blombach, B. Eikmanns, F.R. Bengelsdorf, D. WeusterBotz, P. Durre, Using gas mixtures of $\mathrm{CO}, \mathrm{CO} 2$ and $\mathrm{H}-2$ as microbial substrates: the do's and don'ts of successful technology transfer from laboratory to production scale, Microbial Biotechnology, 11 (2018) 606-625. 


\section{Figure captions}

Fig. 1. A combination of high-resolution microscopic and wide-view macroscopic techniques integrating a state-of-the-art high-speed video camera were utilized for visualization of $i n$-situ gas bubble generation. The different components are (1) AnEMBR, (2) the glycerol immersion tank in which the reactors were placed for image capturing, (3) the long-distance microscopic lens attached to (4) the high-speed video camera, and (5) the data acquisition system.

Fig. 2. Peak volumetric current densities, TMP, and volume of gas for AnEMBRs with (A and B) $2 \mathrm{~m}^{2} / \mathrm{m}^{3}$ (C and D) $4 \mathrm{~m}^{2} / \mathrm{m}^{3}$ and (E and F) $8 \mathrm{~m}^{2} / \mathrm{m}^{3}$ SCSA. Values represent averages of duplicate reactors. Each data point in the plot represents the respective performance of individual batch cycles. Unfilled arrows represent day 35 when the high-speed camera was used to capture the in-situ bubble generation, grey arrows represent day 56 when the Ni-HFMs were sampled for SEM imaging followed by physical/ chemical cleaning, and the black arrows represent day 75 when biomass samples were collected for microbial community analysis. The dashed vertical lines cover the region where unintentional disturbance in $\mathrm{pH}$ was induced.

Fig. 3. The accumulated frequency of all gas bubbles on the cathode surface of the AnEMBRs on day 35(A) and day $55(\mathrm{~B})$.

Fig. 4. Scanning electron micrograph images of (A) virgin Ni-HFM and biofouled Ni-HFM at day 56 for (B) $2 \mathrm{~m}^{2} / \mathrm{m}^{3}$ (C) $4 \mathrm{~m}^{2} / \mathrm{m}^{3}$ and (D) $8 \mathrm{~m}^{2} / \mathrm{m}^{3}$.

Fig. 5. The average relative abundances $(\%)$ of dominant genera ( $\sim 80 \%$ of sequence reads) detected on the cathode of the three AnEMBRs. Others ( $\sim 20 \%$ of sequence reads) represent genera with relative abundance < $1 \%$. g: genus; f: family; o: order. 